\title{
Alterações respiratórias, auditivas e citogenéticas em trabalhadores de um estaleiro no Rio de Janeiro: estudo de caso
}

\author{
Respiratory, audiologic and cytogenetic alterations in \\ workers of a shipyard in Rio de Janeiro: case study
}

\author{
Marcos Massao Murata ${ }^{1 *}$, Isabele Campos Costa-Amaral ${ }^{2 *}$, \\ Leandro Vargas Barreto de Carvalho², Gabriela Rodrigues de Souza ${ }^{3}$, \\ Helena Ramirez Domingos Mainenti², Márcia Aparecida Ribeiro de Carvalho ${ }^{4}$, \\ Daniel Valente², Gilvania Barreto Feitosa Coutinho², \\ Diane Francis Ribeiro do Vale², Camila do Nascimento Rodrigues², \\ Patrícia Canto Ribeiro², Monica Stuck de Oliveira ${ }^{5}$, \\ Marco Antônio Carneiro Menezes², Liliane Reis Teixeira², Ariane Leites Larentis², \\ Maria de Fátima Ramos Moreira², Márcia Soalheiro de Almeida², \\ Hermano Albuquerque de Castro², Rita de Cássia Oliveira da Costa Mattos ${ }^{2}$
}

\footnotetext{
Resumo

Introdução: Este estudo avaliou alterações respiratórias, auditivas e citogenéticas em trabalhadores de um estaleiro em Angra dos Reis, no Estado do Rio de Janeiro, relacionadas à exposição de pintores a solventes, metais e ruído no ambiente de trabalho. Métodos: Foram avaliados função pulmonar, perdas auditivas e índice de reconhecimento da fala, e alterações citogenéticas pelo teste de aberrações cromossômicas. Foram avaliados manganês e chumbo em sangue por espectrometria de absorção atômica. Os indicadores de efeito utilizados para chumbo foram ALAD e ALA-U, determinados por espectrofotometria e cromatografia líquida, respectivamente. Resultados: Seis dos 9 trabalhadores avaliados apresentaram alteração funcional respiratória. Quase $70 \%$ dos 18 trabalhadores avaliados apresentaram audição reduzida, com associação entre PAIR e chumbo em sangue. O percentual médio

${ }^{1}$ Instituto de Biologia Roberto Alcantara Gomes, Universidade do Estado do Rio de Janeiro (UERJ) - Rio de Janeiro (RJ), Brasil.

${ }^{2}$ Centro de Estudos da Saúde do Trabalhador e Ecologia Humana (CESTEH), Escola Nacional de Saúde Pública Sergio Arouca (ENSP), Fundação Oswaldo Cruz (Fiocruz) - Rio de Janeiro (RJ), Brasil.

${ }^{3}$ Centro de Ciências da Saúde (CCS), Instituto de Pesquisas de Produtos Naturais, Universidade Federal do Rio de Janeiro (UFRJ) - Rio de Janeiro, (RJ), Brasil. ${ }^{4}$ Instituto de Estudos em Saúde Coletiva (IESC), Universidade Federal do Rio de Janeiro (UFRJ) - Rio de Janeiro (RJ), Brasil.

${ }^{5}$ Divisão de Dosimetria Externa, Comissão Nacional de Energia Nuclear (CNEN), Instituto de Radioproteção e Dosimetria (IRD) - Rio de Janeiro (RJ), Brasil.

*Estes autores contribuíram igualmente para a realização deste artigo.

Trabalho realizado no Centro de Estudos da Saúde do Trabalhador e Ecologia Humana (CESTEH), na Escola Nacional de Saúde Pública Sergio Arouca (ENSP), Fundação Oswaldo Cruz (Fiocruz) - Rio de Janeiro (RJ), Brasil.

Endereço para correspondência: Ariane Leites Larentis - Centro de Estudos da Saúde do Trabalhador e Ecologia Humana (CESTEH), Escola Nacional de Saúde Pública Sergio Arouca (ENSP), Fundação Oswaldo Cruz (Fiocruz), Rua Leopoldo Bulhões, 1480 - Manguinhos - CEP: 21040-360 - Rio de Janeiro (RJ), Brasil Email: arianelarentis@fiocruz.br; arilarentis@yahoo.com.br

Fonte de financiamento: Edital PAPES IV-Fiocruz-B, gerenciado pelo CNPq (Processo num. 400227/2006-1), que contemplou o projeto "Estudo do impacto da exposição a substâncias químicas como estratégia de investigação do potencial genotóxico e neurotóxico" coordenado por Rita de Cássia Oliveira da Costa Mattos.

Conflito de interesses: nada a declarar.
} 
de recuperação da ALAD foi de 32,9\%, com médias de ALA-U de 1,7 mg g ${ }^{-1}$ creatinina, 4,65 $\mu \mathrm{g} \mathrm{dL}^{-1}$ para Pb-S e $10 \mu \mathrm{g} \mathrm{L}^{-1}$ para $\mathrm{Mn}-\mathrm{S}$ entre os trabalhadores. Foram observadas associações entre ALA-D ativada com Mn-S e com a presença de aberrações cromossômicas. As alterações citogenéticas identificadas foram aneuploidias, separação prematura centromérica e aberrações como formação de anéis, quebras e união de cromátides irmãs. Conclusão: os trabalhadores do estaleiro apresentam alterações que podem ser associadas à exposição ocupacional.

Palavras-chave: Saúde do Trabalhador; função pulmonar; Perda Auditiva Induzida pelo Ruído (PAIR); metais; aberrações cromossômicas.

\begin{abstract}
Introduction: This study evaluated respiratory, audiological and cytogenetic alterations in shipyard workers in Angra dos Reis, state of Rio de Janeiro/Brazil, and their correlation to the occupational exposure of painters to solvents, metals and noise present. Methods: We evaluated pulmonary function, hearing loss and speech recognition, as well as cytogenetic alterations. Indicators of exposure to lead and manganese in blood were evaluated by atomic absorption spectrometry. The determination of ALAD and ALA-U was performed by spectrophotometry and liquid chromatography, respectively. Results: Six of the 9 workers evaluated for lung function had respiratory functional impairment. Almost $70 \%$ of the 18 evaluated workers had reduced hearing, with association between PAIR and values of lead in blood. The average percentage of recovery of ALAD was $32.9 \%$; average was $1.7 \mathrm{mg} \mathrm{g}^{-1} \mathrm{creatinine}$ for ALA-U, $4.65 \mu \mathrm{g} \mathrm{dL}{ }^{-1}$ for Pb-B and $10 \mu \mathrm{g} \mathrm{L}^{-1}$ for Mn-B. We observed associations between activated ALA-D with Mn-B and the presence of chromosomal aberrations. Furthermore, we identified cytogenetic alterations as aneuploidy, premature centromere separation; as well as ring formation, breakage, and sister chromatid union. Conclusion: Shipyard workers presented alterations that can be associated with occupational exposure.
\end{abstract}

Keywords: Worker's health; pulmonary function; noise-induced hearing loss; metals; chromosomal aberrations.

\section{INTRODUÇÃo}

Trabalhadores da indústria naval estão expostos a diferentes substâncias químicas, como solventes presentes nas tintas e vernizes e metais presentes nas poeiras e fumos metálicos. Todos provocam irritações na pele e mucosas, podendo causar dermatites e também distúrbios respiratórios e hepáticos, neoplasias, danos ao sistema nervoso central e pneumoconioses quando inalados ${ }^{1}$.

Outro fator de risco para esses trabalhadores é a exposição a ruídos, em associação a substâncias químicas e temperaturas extremas nos locais confinados do ambiente de trabalho dos estaleiros, indutores de alterações e perdas auditivas ${ }^{1-3}$. O impacto negativo causado pela deficiência auditiva pode repercutir nas relações pessoais, no equilíbrio emocional, nas interações sociais ou ocupacionais do trabalhador. Os efeitos extra-auditivos do ruído podem levar a alterações do aparelho circulatório, digestivo, muscular, sistema nervoso, interferência no sono, distúrbio de equilíbrio (tonturas), problemas psicológicos, dores de cabeça, mudanças repentinas de humor, ansiedade, surgimento de zumbidos e baixo rendimento no trabalho ${ }^{4}$.

Doenças decorrentes de exposições ocupacionais e ambientais têm sido detectadas ao nível celular e molecular para posterior controle e prevenção $0^{5,6}$. Os testes citogenéticos, que utilizam células cultivadas ou retiradas de animais ou indivíduos submetidos a exposições, revelam dois tipos de substâncias mutagênicas: as clastogênicas, que produzem aberrações cromossômicas estruturais, e aquelas que interferem na formação do fuso mitótico, provocando alterações na distribuição dos cromossomos e causando aneuploidias, as aberrações numéricas ${ }^{7-10}$. Biomarcadores citogenéticos em linfócitos periféricos, como as aberrações cromossômicas, têm sido usados para avaliar a exposição ocupacional a agentes carcinogênicos ou mutagênicos ${ }^{10,11}$. Em geral, as mutações cromossômicas são eventos causais no desenvolvimento de neoplasias, e o aumento em danos nos cromossomos pode refletir um aumento no risco de câncer ${ }^{6,8}$. A aplicação da pesquisa de citotoxicidade e genotoxicidade em estudos populacionais com exposições a substâncias clastrogênicas pode contribuir para o conhecimento do potencial carcinogênico das substâncias em humanos ${ }^{12}$.

Considerando a exposição ocupacional na indústria naval, há mais de quinze anos o Centro de Estudos da Saúde do Trabalhador e Ecologia Humana (CESTEH) da Escola Nacional de Saúde Pública Sergio Arouca (ENSP), Fundação Oswaldo Cruz, tem desenvolvido alguns estudos de avaliação da exposição ocupacional em estaleiros no Estado do Rio de Janeiro ${ }^{1,13-16}$. Nesse contexto, encontra-se este trabalho, que teve como objetivo avaliar a exposição de pintores de um estaleiro a solventes e metais, e identificar possíveis alterações respiratórias, auditivas e citogenéticas, caracterizadas pela ocorrência de aberrações cromossômicas.

\section{MÉTODOS}

O grupo exposto avaliado neste estudo foi composto por uma amostra de conveniência de pintores $(n=18)$ de um estaleiro em Angra dos Reis, RJ. Tratou-se de um estudo observacional transversal, tendo como critérios de inclusão: trabalhadores em contato com a tinta utilizada no estaleiro, idade acima de 18 anos e tempo de trabalho mínimo de 6 meses no estaleiro. Excluíram-se os trabalhadores que apresentaram comorbidades preexistentes à entrada no estaleiro e durante os seis primeiros meses de trabalho assim como aqueles que usavam medicamentos antineoplásicos e com realização de exame de raio-X no último mês. Um questionário solicitou informações sociodemográficas e sobre consumo de álcool, fumo e hábitos de lazer, bem como uso de medicamentos, exames realizados e doenças preexistentes. 
Estes dados foram importantes para a identificação de fatores de confundimento na análise citogenética. Nenhum indivíduo foi excluído do estudo devido ao hábito de fumar ou ingerir bebida alcoólica. O estudo foi aprovado pelo Comitê de Ética em Pesquisa da Fiocruz sob o número CAAE: 0037.0.031.000-06, e os participantes assinaram termo de consentimento.

Os trabalhadores realizaram os testes audiológicos, pneumológicos e dos indicadores biológicos no CESTEH/ENSP/Fiocruz, RJ. Os exames hematológicos foram realizados pelo Laboratório de Análises Clínicas do Instituto Nacional de Infectologia Evandro Chagas/Fiocruz, RJ.

\section{Avaliação pneumológica}

Os exames radiográficos de tórax e espirometria tiveram a finalidade de avaliar o grau de acometimento dos trabalhadores. Os padrões da International Labour Organization (ILO) nortearam a técnica radiológica, cuja classificação se deu da seguinte maneira: 0/- ou 0/0 - raio-X normal; $0 / 1$ - suspeito de silicose; $1 / 0$ ou $1 / 1$ ou $1 / 2$ - silicose grau $1 ; 2 / 1$ ou $/ 2$ ou $2 / 3$ - silicose grau $2 ; 3 / 2$ ou $3 / 3$ ou $3 /+-$ silicose grau 3 (p;q;r representam lesões nodulares e s; $;$ u representam lesões lineares). O teste espirométrico seguiu a American Thoracic Society (ATS), que consiste numa expiração forçada até o limite do volume de reserva expiratória, após inspiração máxima. A espirometria avalia a função ventilatória, refletindo dados sobre fluxos e volumes pulmonares, sendo a forma de avaliação funcional mais comum, rápida, de fácil execução e baixo custo. É um teste que auxilia na prevenção, além de permitir o diagnóstico e a quantificação dos distúrbios ventilatórios, tendo importante papel na pneumologia ocupacional ${ }^{14}$.

\section{Avaliação audiológica}

A identificação dos riscos e a avaliação dos níveis de pressão sonora existentes no ambiente de trabalho foram interpretados e analisados através da norma determinada pela NBR 10152, Normas Regulamentadoras ${ }^{17}$ do Ministério do Trabalho e Instrução Normativa do INSS ${ }^{18}$ No 608 para perda auditiva neurossensorial. As pesquisas audiológicas seguiram as etapas de inspeção do meato acústico externo, para verificar a possibilidade de prosseguimento do exame audiológico; exame auditivo básico, através da Audiomeria Tonal Liminar e Vocal e da Imitanciometria; e provas supraliminares (Sisi e ToneDecay Test), utilizadas para estabelecer o diagnóstico diferencial da perda auditiva. A anamnese audiológica investigou as correlações entre a exposição/vulnerabilidade ao ruído e/ou substâncias químicas. Os indivíduos que apresentaram alterações foram encaminhados para avaliação otorrinolaringológica e registrados para acompanhamento e monitoramento. Protocolos de investigação registraram a história audiológica pregressa e agravos auditivos e extra-auditivos. As interferências na fala foram investigadas através da determinação do Índice de Reconhecimento da Fala (IRF) em ambas as orelhas.

\section{Análise de indicadores biológicos de exposição a metais}

As concentrações de chumbo ( $\mathrm{Pb}-\mathrm{S}$ ) e manganês em sangue (Mn-S) foram determinadas no espectrômetro de absorção atômica Zeeman 5100, com tubos e plataformas de grafite pirolítico (Perkin-Elmer, Norwalk, CT USA) e medidas de absorvância integrada (área de pico) ${ }^{19}$.

Os indicadores de efeito ácido delta-aminolevulínico desidratase (ALAD) e ácido delta-aminolevulínico na urina (ALA-U) foram determinados conforme descritos por Murata ${ }^{15}$. A determinação da atividade de ALAD em eritrócitos foi realizada por espectrofotometria em $555 \mathrm{~nm}$. O percentual de recuperação da atividade da ALAD (\% ALAD) corresponde à diferença entre a enzima ativada e a inativada, calculada considerando a atividade da enzima ativada como $100 \%$ e Índice Biológico Máximo (IBM) de até $40 \%{ }^{20}$. As amostras de urina foram analisadas por cromatografia líquida em coluna de fase reversa C18.

\section{Avaliação de aberrações cromossômicas (AC)}

Amostras de sangue, coletadas em tubos heparinizados e acondicionados em gelo, foram inseridas em meio de cultura Ham's F-10, suplementados com $20 \%$ de soro bovino fetal e $4 \%$ fitohematoglutinina. As lâminas foram preparadas segundo descrição detalhada em Murata ${ }^{15}$. O espalhamento das metáfases na lâmina ao microscópio óptico, sob contraste de fase, foi realizado em triplicata e com aumento de 1000X, usando o programa Metafer. Cada célula metafásica foi analisada contando-se o número de figuras (presença de fragmentos acêntricos), observando a morfologia dos cromossomos e a presença de aberrações estruturais do tipo cromatídica e cromossômica.

\section{Análise estatística}

$\mathrm{Na}$ análise descritiva foram calculadas médias, desvios padrão e frequência percentual de distribuição. A normalidade da distribuição das variáveis contínuas foi testada por meio do teste Kolmogorov-Smirnov. Para comparação de variáveis com distribuição normal utilizaram-se os testes paramétricos $t$ de Student ou ANOVA, e para variáveis com distribuição não normal utilizaram-se os testes não paramétricos Mann-Whitney (U) ou Kruskal-Wallis (H). Testes de correlação de Person (r) e de Spearman (rho) foram realizados para as variáveis contínuas com distribuição normal e não normal, respectivamente. $\mathrm{O}$ teste de Mann-Whitney $U$ foi empregado para avaliar a relação entre os trabalhadores com laudo indicativo de PAIR e as concentrações de metais em sangue. O teste Chi-Quadrado para uma única variável foi empregado para comparar as alterações citogenéticas 
com os de uma população considerada normal, descrita na literatura ${ }^{21}$. A variável idade foi utilizada como variável controle. Foi adotado nível de significância de $5 \%$ em todas as análises. Os dados foram analisados com auxílio do programa SPSS for Windows versão 20.0 .

\section{RESULTADOS}

Participaram do estudo 18 trabalhadores do sexo masculino, que apresentavam quadro alérgico, sintomas de pele e pulmonares, sendo praticamente $90 \%$ pintores ou exerciam atividades relacionadas à pintura de navios e plataformas petrolíferas. O tempo de trabalho na atividade variava de 2 a 30 anos (média = 14 anos), com carga horária de trabalho semanal de 44 h. Entre os trabalhadores, $65 \%$ possuíam ensino fundamental incompleto, $20 \%$ informaram ser fumantes (média de 25 cigarros/dia) e 65\% relataram ingerir bebida alcoólica socialmente.

Os equipamentos de proteção individual (EPIs) foram descritos como de uso frequente. Os trabalhadores não levavam o uniforme para casa e utilizavam macacão descartável em alguns processos de pintura. Foram identificados vários trabalhadores com dermatoses, embora fizessem uso de EPIs, tais como macacão hidrorrepelente descartável, máscara (carvão), luva de cano longo, protetor auricular, botas e óculos.

Os trabalhadores relataram nunca ter usado medicamentos antineoplásicos e haver feito exames de raio-X, em média, há mais de um mês. No entanto, vários citaram o uso de medicamentos para alergias respiratórias e dermatológicas. As avaliações clínicas constataram que a grande maioria destes trabalhadores apresentava dermatoses, pneumopatias e problemas auditivos relacionados às atividades laborais. Foram relatados casos de adoecimento e exposição no processo de pintura, bem como a relação de conflitos entre a empresa e o INSS quanto aos encaminhamentos dos casos dos trabalhadores com doenças ocupacionais.

Do total de nove trabalhadores avaliados para função pulmonar, seis se encontravam com alteração funcional respiratória (distúrbio ventilatório obstrutivo). Neste grupo, foram diagnosticados quatro casos de asma, além de um caso de silicose e três com radiologia suspeita. Apesar do número de trabalhadores avaliados não ser grande, é possível observar que a maioria apresentava sérios problemas pulmonares relacionados ao trabalho. Essas alterações podem representar os danos gerados pelas exposições ocupacionais, como tintas entre pintores, semelhantes à asma ocupacional com padrão de obstrução. Entre casos analisados, apenas três apresentaram exames funcionais e radiológicos normais.

Com relação à avaliação audiológica, somente 50\% dos 18 trabalhadores avaliados consideravam sua audição normal; $30 \%$ pouco reduzida e $20 \%$ muito reduzida. O grupo de trabalhadores apresentou $67 \%$ de perda auditiva induzida pelo ruído (PAIR) com alterações extra-auditivas, como a presença de zumbido, relatada por $60 \%$ dos trabalhadores (5\% sempre, $25 \%$ frequentemente e $30 \%$ raramente), e tontura relatada por $65 \%$ deles. Com relação aos impactos psicossociais avaliados pelo questionário decorrentes da perda auditiva, 10\% dos trabalhadores relataram que sua audição interferia no seu desempenho, 15\% na sua vida social e 35\% nas oportunidades. Foram também avaliados os Índices de Reconhecimento da Fala (IRF) para ambas as orelhas de 18 trabalhadores, sendo obtidos os resultados de $91,8 \pm 7,7$ para orelha direita e $92,8 \pm 12,7$ para orelha esquerda. A Tabela 1 relaciona os achados audiológicos com os valores de $\mathrm{Pb}-\mathrm{S}$ e Mn-S. Além de encontrar trabalhadores com indicativos de PAIR, foi observada uma diferença significativa entre as médias de chumbo em sangue nos trabalhadores com laudo indicativo de PAIR e aqueles com limites aceitáveis.

A Tabela 2 apresenta os valores dos indicadores biológicos de exposição ao $\mathrm{Pb}$ e Mn. Quatro trabalhadores (22,2\%) tiveram o percentual de recuperação da enzima ALAD (média = 41,6\%) igual ou acima do IBM de $40 \%{ }^{20}$. A média para todo o grupo foi de $32,9 \%$. Os níveis de ALA-U apresentaram média de $1,7 \mathrm{mg} \mathrm{g}^{-1}$ creatinina no grupo exposto, e um trabalhador deste grupo apresentou nível igual a 4,2 $\mathrm{mg} \mathrm{g}^{-1}$ creatinina, além de uma concentração $\mathrm{Pb}-\mathrm{S}$ de $7,6 \mu \mathrm{g} \mathrm{dL} \mathrm{dL}^{-1}$. O nível de chumbo no sangue dos trabalhadores comportou-se de forma semelhante ao

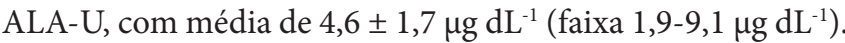

Tabela 1. Resultados obtidos de perda auditiva neurossensorial dos trabalhadores do estaleiro e relação com exposição a chumbo e manganês $(n=18)$

\begin{tabular}{cccc}
$\begin{array}{c}\text { Resultado do Laudo } \\
\text { Audiológico }\end{array}$ & $\mathbf{n}$ & $\begin{array}{c}\mathbf{P b}-\mathbf{S} \\
\left(\boldsymbol{\mu g} \mathbf{d L} \mathbf{L}^{-1}\right)\end{array}$ & $\begin{array}{c}\mathbf{M n}-\mathbf{S} \\
\left(\boldsymbol{\mu g} \mathbf{~ L}^{-1}\right)\end{array}$ \\
\hline $\begin{array}{c}\text { Dentro dos limites aceitáveis } \\
\text { PAIR }\end{array}$ & 6 & $4,0 \pm 0,8$ & $9,3 \pm 2,2$ \\
\hline
\end{tabular}

$\mathrm{Pb}-\mathrm{S}$ (chumbo em sangue) e Mn-S (manganês em sangue) avaliados por média \pm desvio padrão; ${ }^{\star} \mathrm{p}<0,01$ dentro do próprio grupo para o teste de Mann-Whitney U

Tabela 2. Indicadores biológicos de exposição aos metais, com seus valores médios, mínimos e máximos $(n=18)$

\begin{tabular}{|c|c|c|c|c|}
\hline Indicadores & Médias & $\begin{array}{l}\text { Desvio } \\
\text { padrão }\end{array}$ & Mínimo & Máximo \\
\hline ALAD inativada ${ }^{1}$ & 1432 & 385 & 930 & 2299 \\
\hline ALAD ativada ${ }^{1}$ & 2069 & 488 & 1433 & 3195 \\
\hline$\% \operatorname{rec} \mathrm{ALAD}^{2}$ & 32,9 & 9,6 & 1,1 & 43,5 \\
\hline ALA-U (mg g ${ }^{-1}$ creat $)^{3}$ & 1,7 & 0,8 & 0,8 & 4,2 \\
\hline $\mathrm{Pb}-\mathrm{S}\left(\mu \mathrm{g} \mathrm{dL} \mathrm{L}^{-1}\right)$ & 4,65 & 1,7 & 1,9 & 9,1 \\
\hline $\mathrm{Mn}-\mathrm{S}\left(\mu \mathrm{g} \mathrm{L}^{-1}\right)$ & 10,0 & 2,6 & 5,5 & 15,3 \\
\hline
\end{tabular}

${ }^{1}$ atividade de ALAD expressa em $\mu \mathrm{mol}$ de profobilinogênio hora ${ }^{-1} \mathrm{~L}^{-1}$ de hematócrito a $37^{\circ} \mathrm{C} ;{ }^{2} \%$ de recuperação (\% rec) de ALAD corresponde ao $\%$ da diferença entre a atividade da enzima inativada e a ativada (após tratamento com ditiotreitol); ${ }^{3}$ atividade de ALA-U corrigida por creatinina ( $\left.\mathrm{mg} \mathrm{g}^{-1} \mathrm{creat}\right)$ 
Com relação à concentração de Mn-S, o grupo exposto apresentou média de $10 \pm 2,6 \mu \mathrm{g} \mathrm{L}^{-1}$ (faixa 5,5-15,3 $\mu \mathrm{g} \mathrm{L} \mathrm{L}^{-1}$ ).

Na observação de alterações citogenéticas, as amostras de 13 trabalhadores do estaleiro foram analisadas. As alterações observadas foram aneuploidias, separação prematura centromérica (SPC) e aberrações cromossômicas (AC), como anel, quebras, união de cromátides irmãs, mostradas na Tabela 3 . Foram avaliadas 808 metáfases, com total de 630 válidas, e encontradas alterações como anéis (2), quebras cromatídica (1) e cromossômica (1). Com relação às SPCs, um trabalhador apresentou valor excessivo, distante da média do grupo exposto. Este indivíduo, que já apresentava distúrbios hematológicos importantes como leucopenia e valores elevados da célula bastão, foi encaminhado para acompanhamento clínico mais rigoroso. Foram encontradas correlações positivas e significativas entre aneuploidias e SPCs $(r=0,463 ; p=0,013)$, bem como entre aneuploidias e ACs $(\mathrm{r}=0,533 ; \mathrm{p}=0,003)$.

A Tabela 4 apresenta a comparação entre os resultados de alterações citogenéticas encontradas neste estudo com os mesmos testes avaliados em uma população considerada normal, descrita na literatura ${ }^{21}$. Os valores do $\chi 2$ encontrados para aneuploidia e SPC foram 100,01 e 321,0 ( $<<0,001)$, respectivamente. Com relação a ACs, os valores encontrados foram $\chi 2=8,61$ para o grupo de trabalhadores do estaleiro e $\chi^{2}=13,61$ para todos os indivíduos $(\mathrm{p}<0,01)$.

Tabela 3. Indicadores citogenéticos nas populações dos trabalhadores do estaleiro $(n=13)$

\begin{tabular}{lcccc} 
Indicadores citogenéticos & Média & $\begin{array}{c}\text { Desvio } \\
\text { padrão }\end{array}$ & Mínimo & Máximo \\
\hline $\begin{array}{l}\text { Aneuploidias } \\
\text { Separação Prematura }\end{array}$ & 5,7 & 4,0 & 0 & 14 \\
$\begin{array}{l}\text { Centromérica } \\
\text { Aberrações }\end{array}$ & 3,5 & 6,5 & 0 & 24 \\
Cromossômicas & 0,46 & 0,97 & 0 & 3 \\
\hline
\end{tabular}

Tabela 4. Frequências das alterações citogenéticas esperadas e observadas no grupo de trabalhadores do estaleiro $(n=13)$ e em todos os indivíduos $(n=18)$ em linfócitos

\begin{tabular}{ccccc} 
Grupo & $\begin{array}{c}\text { Frequência } \\
\text { observada }\end{array}$ & $\begin{array}{c}\text { Proporção } \\
\text { de } \\
\text { referência }\end{array}$ & $\begin{array}{c}\text { Frequência } \\
\text { esperada }\end{array}$ & $\chi^{2}$ \\
s/Aneup & 525 & 9423 & 587,8 & $100,01^{* * *}$ \\
c/Aneup & 105 & 677 & 42,2 & \\
Total & 630 & 10100 & 630 & \\
s/SPC & 588 & 10029 & 625,6 & $321,0^{* * *}$ \\
c/SPC & 42 & 71 & 4,4 & \\
Total & 630 & 10100 & 630 & \\
s/AC & 623 & 10061 & 627,6 & $8,61^{* *}$ \\
c/AC & 7 & 39 & 2,4 & \\
Total & 630 & 10100 & 630 & \\
\hline
\end{tabular}

${ }^{* *} \mathrm{p}<0,01 ; \chi^{2}$ crítico $=6,64 ;{ }^{* *} \mathrm{p}<0,001 ; \chi^{2}$ crítico $=10,83$
A Tabela 5 mostra os resultados dos indicadores respiratórios, audiológicos e citogenéticos para cada um dos 18 trabalhadores do estaleiro avaliados no estudo. A avaliação conjunta de todos os indicadores do grupo de trabalhadores, controlada por idade e tabagismo, mostrou correlação significativa entre os resultados de ALA-D ativada e Mn-S ( $r=0,756 ; \mathrm{p}=0,030)$ e entre IRF-OD e Pb-S ( $r=-0,621 ; p=0,006)$. A correlação entre os resultados de ALA-D ativada e a presença de aberrações cromossômicas ficou próxima da significância estatística $(r=-0,677 ; p=0,065)$, assim como a correlação entre os resultados de IRF-OD e SPC $(r=0,536 ; p=0,059)$. Os demais indicadores não apresentaram correlação estatisticamente significativa.

\section{DISCUSSÃO}

Neste estudo, foram avaliadas alterações respiratórias, auditivas e citogenéticas em pintores de um estaleiro em Angra dos Reis, expostos a solventes e metais. Compostos de metais, como o chumbo e manganês, são muito usados como pigmentos para tintas ${ }^{22}$. Recentemente, o limite máximo permitido de chumbo no Brasil, para tintas imobiliárias, de uso infantil e escolar, vernizes e materiais similares de revestimento de superfícies, foi limitado a uma concentração igual ou superior a $0,06 \%(\mathrm{p} / \mathrm{p})$ (Lei ${ }^{\circ} 11.762 / 2008$ ), que ainda pode ser considerado alto em comparação com outros países, onde o chumbo é proibido em tintas domésticas. Por outro lado, fumaça, poeira e aerossóis de processamento metalúrgico, fundição de aço, soldagem e corte de metal liberam óxido de manganês para o ambiente ${ }^{23}$.

Diversos tipos de solventes podem ser adicionados às tintas com o objetivo de torná-las mais fluidas. Tolueno e benzeno estão entre os mais utilizados por trabalhadores ocupacionalmente expostos a solventes aromáticos e considerados genotóxicos ${ }^{11,24-26}$. O benzeno é considerado um carcinógeno humano, com atividade clastogênica ${ }^{25}$. O tolueno é considerado ototóxico, com evidências de interação com ruído; já o potencial ototóxico do benzeno pode ser considerado inconclusivo ${ }^{27}$. De acordo com os protocolos das Normas e Manuais Técnicos do Ministério da Saúde relacionados à exposição ocupacional ao benzeno ${ }^{28,29}$, as avaliações de PAIR e de alterações cromossômicas, entre outras, devem ser realizadas em trabalhadores que utilizam este composto em seu processo de trabalho. A composição dos pigmentos, solventes e tintas mudam conforme a necessidade do processo. Desse modo, os trabalhadores estão sempre expostos a diferentes substâncias químicas. Em geral, o maior risco na exposição às substâncias químicas se deve a processos que envolvam a formação de aerossóis, pós e poeiras, e fumos, daí a necessidade de avaliar provas de função pulmonar na investigação de doenças ocupacionais respiratórias relacionados ao processo de trabalho investigado, assim como no estabelecimento de incapacidade em pacientes com pneumoconiose $\mathrm{e}^{14}$. 


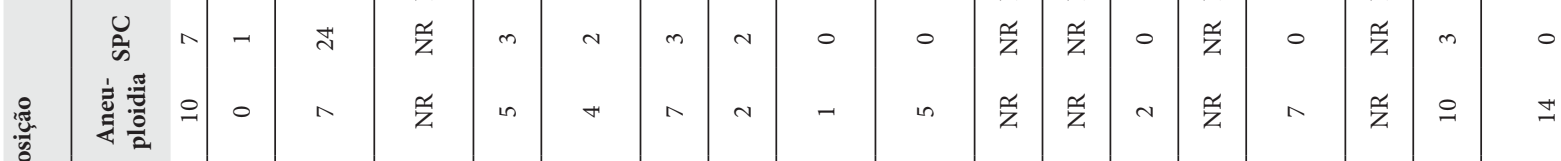

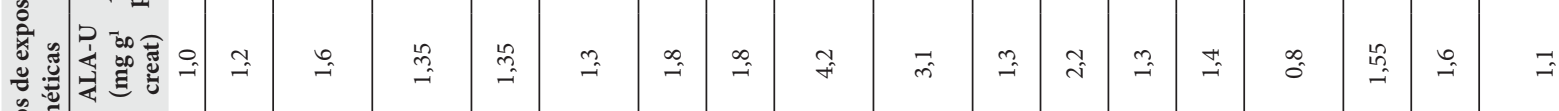

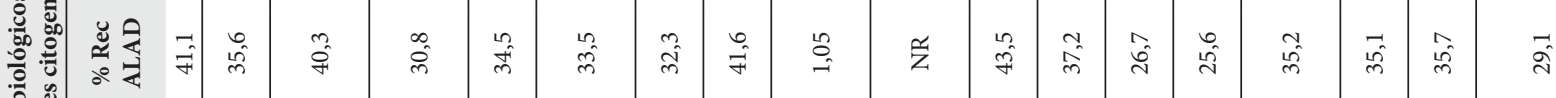

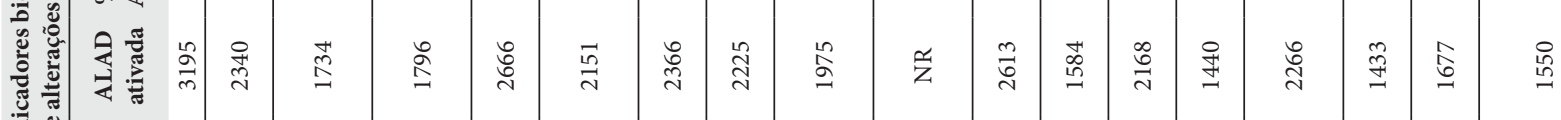

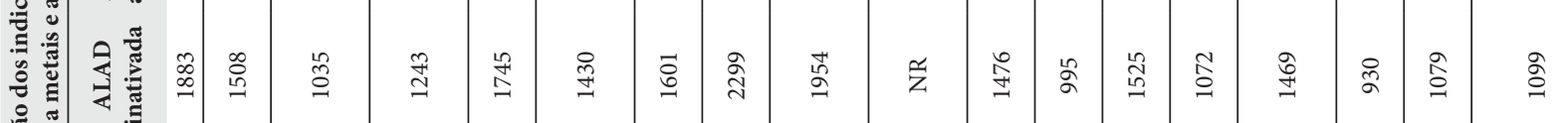

采

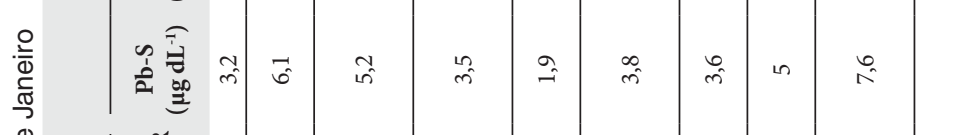

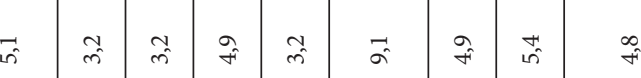

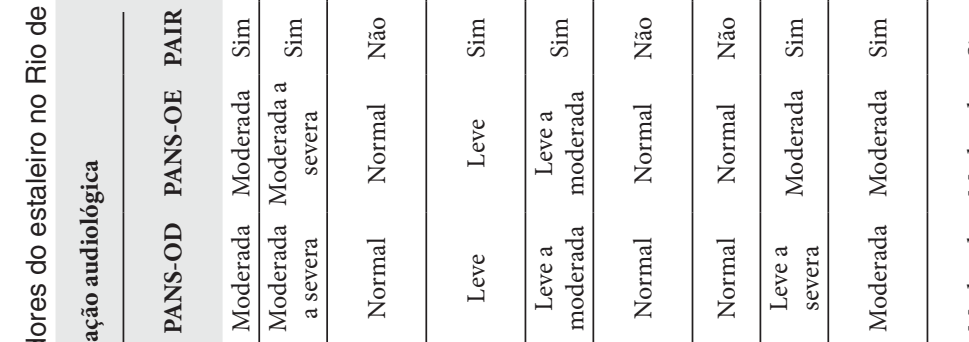

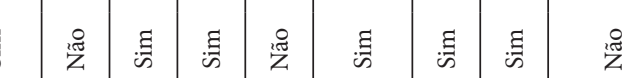

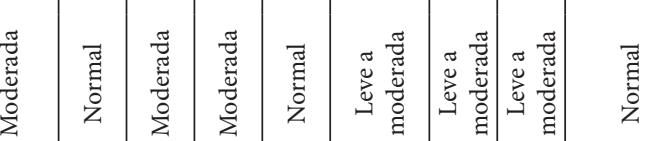

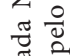

호 원

胥

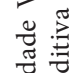

莡专

论

คิ

芑芯

品

预点

zั

苞

氙

蛋要

苞

造

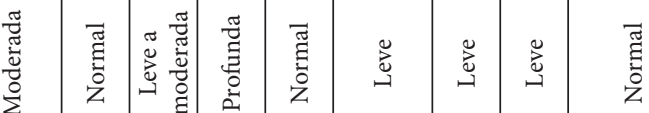

乙

岂焉

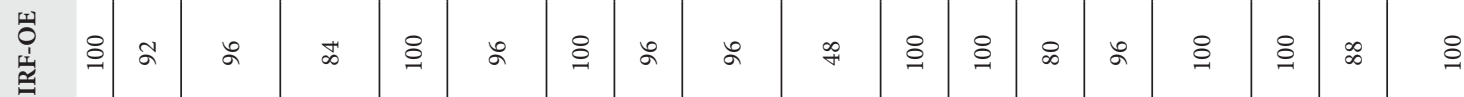

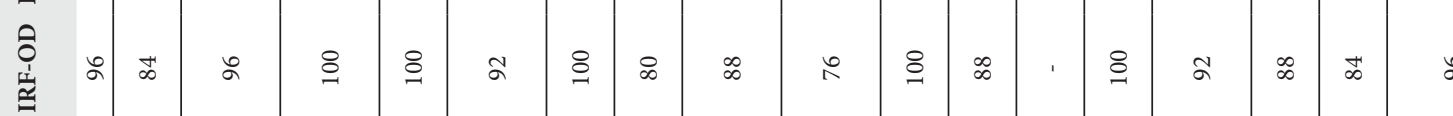

要䨪

劳 됭

$\frac{\infty}{0}$

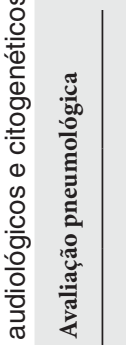

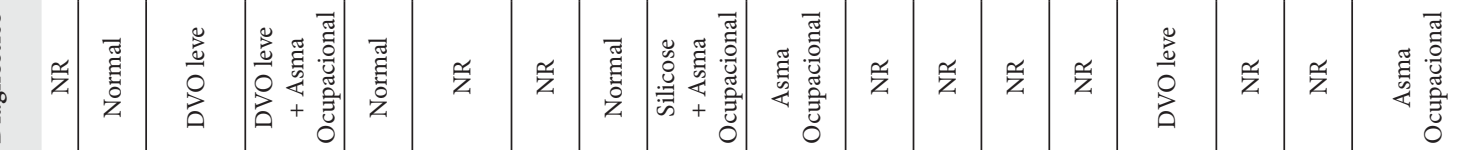

范

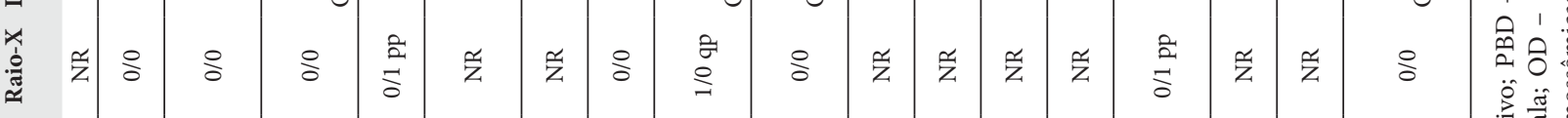

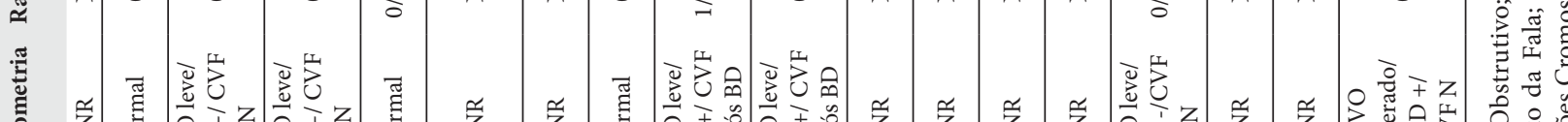

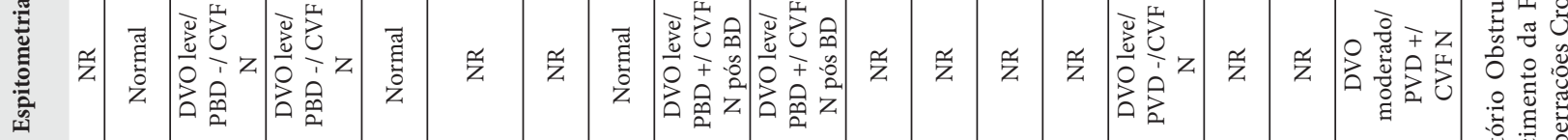

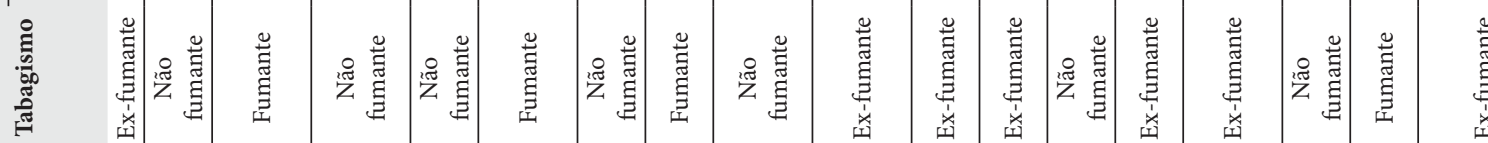

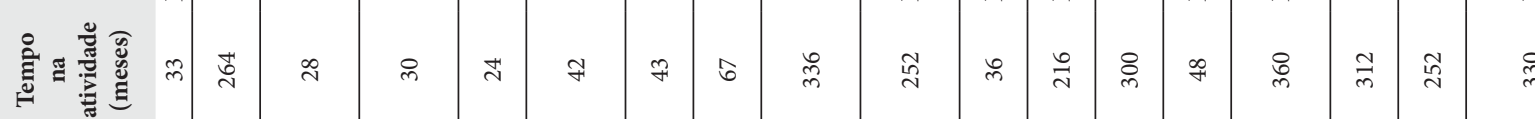

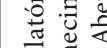

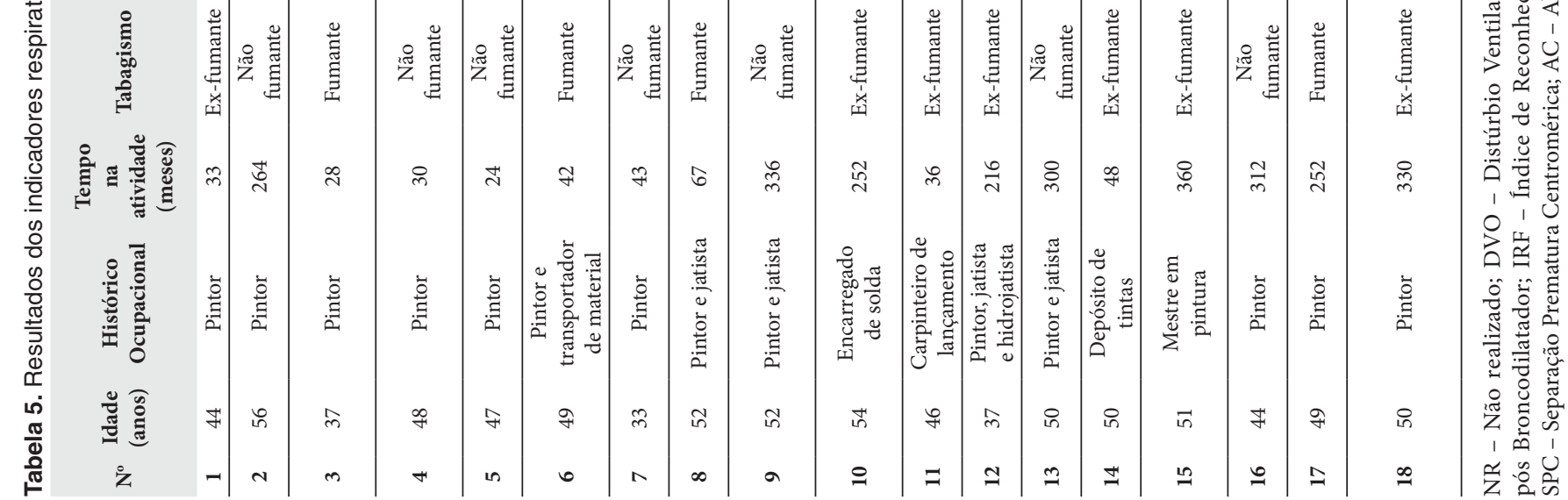


Dos 18 participantes, apenas uma parte dos trabalhadores aceitaram participar das avaliações pneumológicas, audiológicas, de exposição a metais e aberrações cromossômicas. Esse grupo de trabalhadores pode ter continuado no estudo, devido a problemas de saúde que não haviam sido investigados anteriormente, mas não podem representar o estado de saúde de todos os trabalhadores de estaleiros (viés de seleção). Não foram encontrados na literatura trabalhos que avaliem concomitantemente alterações citogenéticas e auditivas em trabalhadores expostos a solventes como benzeno e tolueno e metais, embora estas avaliações sejam indicadas nas Normas e Manuais Técnicos do Ministério da Saúde, tendo em vista as dificuldades de sua avaliação. Os testes de aberrações cromossômicas foram realizados em um número reduzido de pintores, dada a dificuldade técnica, que apesar de seu enorme potencial na saúde ocupacional e biomonitoramento, poucos são os trabalhos que os empregam, especialmente no Brasil.

Há na literatura alguns estudos em populações brasileiras que mostram a exposição ocupacional de ruído associado a substâncias químicas, como metais pesados, agrotóxicos/inseticidas, solventes e gases (como monóxido de carbono) ${ }^{30-41}$. As interações desses agentes com o ruído devem ser avaliadas cuidadosamente e incluídas nos programas de prevenção à perda auditiva ${ }^{42,43}$.

O grupo de trabalhadores do estaleiro apresentou interferência do ruído na vida laboral e social. Os trabalhadores apresentaram IRF menores para as duas orelhas, evidenciando influências na fala. A percepção dos sons da fala depende de um sistema auditivo perceptual periférico íntegro, o que não ocorre nas perdas auditivas neurossensoriais investigadas neste estudo. Esse tipo de comprometimento auditivo afeta inicialmente frequências altas, levando a uma dificuldade acentuada na percepção da inteligibilidade da fala. Nos casos de perda auditiva induzida por ruído, são comuns as dificuldades de diferenciar os sons da fala e de compreender uma mensagem, que aumentam consideravelmente quando o trabalhador encontra-se em ambiente ruidoso. À medida que a lesão auditiva avança para frequências mais graves, afeta a percepção de palavras faladas de longe. Circunstâncias ambientais desfavoráveis reduzem a capacidade de distinguir detalhes dos sons da fala, pois o mascaramento e a reverberação ambientais afetam os mecanismos de compensação, reduzindo a inteligibilidade. O ruído, por seus efeitos de mascaramento sobre a palavra ou outros sinais sonoros úteis como alarmes, pode dar origem a acidentes profissionais ${ }^{42}$.

Os efeitos do chumbo sobre o sistema auditivo vêm sendo investigados por alguns pesquisadores ${ }^{30,44,45}$. Existem inúmeros estudos acerca dos efeitos da contaminação pelo chumbo no organismo, demonstrando que múltiplos órgãos podem ser afetados. Entretanto, pesquisas sobre os efeitos do chumbo no sistema auditivo e exposição simultânea ao chumbo e outros agentes, como o ruído, são escassas, além de apresentarem resultados contraditórios ${ }^{30,44}$. Correlações significativas entre os níveis de chumbo em sangue e os limiares auditivos foram descritas em diferentes exposições ocupacionais ${ }^{44}$. No atual estudo com trabalhadores do estaleiro, foi verificada associação entre a perda auditiva e a presença de chumbo no sangue, embora os resultados de $\mathrm{Pb}-\mathrm{S}$ encontrados para os trabalhadores estejam abaixo dos limites adotado pela NR-7 $7^{46}$, que estabelece valor de referência de $40 \mu \mathrm{g} \mathrm{dL}^{-1}$ para pessoas não expostas, e índice biológico máximo permitido (IBMP) de $60 \mu \mathrm{g} \mathrm{dL}^{-1}$, limite para pessoas expostas ocupacionalmente, pois acima dele são verificados efeitos na saúde. Os limites indicados por algumas agências americanas, como $\mathrm{OSHA}^{47}$ e $\mathrm{ACGIH}^{48}$, são também altos, visto que o valor de referência para chumbo em sangue é de $30 \mu \mathrm{g} \mathrm{dL}^{-1}$. Entretanto, estes valores estão muito longe de serem seguros, pois a inibição da ALAD já ocorre em torno de $20 \mu \mathrm{g} \mathrm{dL}^{-123}$. A NIOSH${ }^{49}$ reconhece que os níveis de chumbo no sangue para adultos são elevados e recomenda que sejam reduzidos para $<25 \mu \mathrm{g} \mathrm{dL}^{-1}$. Ainda assim, os resultados deste trabalho indicam perda auditiva nos trabalhadores para valores de $\mathrm{Pb}$-S muito inferiores aos indicados nestas legislações.

Ainda há um hiato no que se refere aos limites biológicos seguros para a exposição ao $\mathrm{Mn}^{50}$. O uso de $\mathrm{Mn}$-S é aconselhado para estudos de base populacional, e não no monitoramento biológico individual ${ }^{19,50}$. Na Comunidade Europeia foram encontrados níveis de Mn variando de 7,1-10,5 $\mu \mathrm{g} \mathrm{L}^{-1} \mathrm{em}$ sangue e 0,12-1,9 $\mu \mathrm{g} \mathrm{L}^{-1} \mathrm{em}$ urina na população em geral ${ }^{51}$. Uma pesquisa realizada com trabalhadores de uma oficina de tratamento de superfície para recuperação de armamento encontrou teores de Mn-S variando de 4,34-7,50 $\mu \mathrm{g} \mathrm{L} \mathrm{L}^{-11}$. Outro estudo determinou a concentração de Mn-S de 23 trabalhadores de um estaleiro, também em Angra dos Reis/RJ ${ }^{16}$, encontrando valores mínimo e máximo iguais a 4,92 e 22,04 $\mu \mathrm{g} \mathrm{L} \mathrm{L}^{-1}$, respectivamente. A legislação brasileira não possui limites biológicos deste metal, visto que a NR- $7^{46}$ não os cita. No entanto, os valores de referência da $\mathrm{ATSDR}^{52}$ para $\mathrm{Mn}-\mathrm{S}$ em grupos não expostos ocupacionalmente encontram-se na faixa de 4-15 $\mu \mathrm{g} \mathrm{L} \mathrm{L}^{-1}$.

A avaliação do grupo exposto apontou para um elevado índice de casos de pneumonia. Os alvos primários, após exposições crônicas ao manganês e seus compostos, são os pulmões e o cérebro. Alta incidência de pneumonia foi associada com concentrações acima de $210 \mu \mathrm{g} \mathrm{L}^{-1}$ de manganês no ambiente de trabalho ${ }^{22}$. Uma avaliação mais precisa deve ser conduzida para se assegurar que o grupo de trabalhadores do estaleiro seja considerado ocupacionalmente exposto ao Mn.

Com relação aos resultados dos indicadores de efeito, embora os níveis de ALA-U obtidos no grupo de trabalhadores do estaleiro estivessem abaixo do valor empregado como referência (4,5 $\mathrm{mg} \mathrm{g}^{-1}$ creatinina) pela NR- $7^{46}$, um trabalhador desse grupo apresentou um resultado próximo desse valor limite 
(4,2 $\mathrm{mg} \mathrm{g}^{-1}$ creatinina) e concentração $\mathrm{Pb}-\mathrm{S}$ de $7,6 \mu \mathrm{g} \mathrm{dL} \mathrm{dL}^{-1}$, podendo indicar uma exposição ocupacional ou ambiental ao chumbo ${ }^{53}$.

Estudos de mortalidade por câncer em humanos, tentando correlacionar exposição crônica a chumbo e ocorrência de câncer, têm mostrado um moderado poder de predição específica do risco relacionado ao metal, devido às populações expostas sofrerem diversas outras exposições sobrepostas a carcinógenos, como arsênio, cromo hexavalente, cádmio, além dos hidrocarbonetos aromáticos e tabagismo ${ }^{23}$. A IARC ${ }^{54}$ classifica os compostos inorgânicos de chumbo no grupo $2 \mathrm{~A}$, i.e., provavelmente carcinogênico para humanos, visto que o grau de evidência de carcinogenicidade em humanos é quase suficiente, enquanto e as evidências já são comprovadas em animais. Os resultados encontrados mostraram que os trabalhadores do estaleiro se encontravam expostos ocupacionalmente aos compostos inorgânicos de chumbo.

Alterações cromossômicas como as observadas nos trabalhadores do estaleiro são biomarcadores bem estabelecidos de exposição ocupacional e ambiental a agentes genotóxicos. Tem sido proposto que a frequência de aberrações cromossômicas em linfócitos de sangue periférico possa servir não só como indicador de exposição a mutagênicos, como também indicador de danos genéticos com relevância no processo carcinogênico ${ }^{6,8}$. Por exemplo, trabalhadores expostos ocupacionalmente a substâncias como benzeno e outros solventes orgânicos têm uma maior incidência de aberrações cromossômicas em comparação à população em geral ${ }^{8}$. Essas alterações citogenéticas são também utilizadas como indicadores de efeitos precoces nos estudos de monitoramento biológico em populações expostas a diversos agentes genotóxicos ${ }^{7,10}$, como verificado em (poucos) estudos brasileiros ${ }^{55-65}$

Neste estudo com os trabalhadores do estaleiro, a aneuploidia, avaliada no teste de aberrações cromossômicas, apresentou alta correlação com as outras alterações citogenéticas. As alterações citogenéticas apresentadas foram diferentes daquelas encontradas no grupo controle da pesquisa de Major et al. ${ }^{21}$, cuja frequência de aneuploidias foi de 6,7\%. O presente estudo encontrou $16,7 \%$ no grupo de trabalhadores do estaleiro, podendo ser uma evidência de que esses trabalhadores estavam expostos ocupacionalmente a substâncias clastogênicas. Vários estudos indicam que a instabilidade cromossômica, que pode significar falhas na separação mitótica, seja a causa principal da aneuploidia, característica comum das células cancerosas. A presença desses cromossomos extras (na maioria das vezes, essas células cancerosas possuem mais de 100) faz com que as células sejam instáveis, causando alterações severas no controle da divisão celular. A instabilidade cromossômica é uma característica comum em tumores de humanos, podendo resultar de defeitos na segregação cromossômica, na estabilidade do telômero, na regulação do ciclo celular, ou no reparo de DNA. Em células normais, vários sistemas corrigem os acoplamentos anômalos entre os cinetócoros e o fuso mitótico ${ }^{6,9,15}$.

Major et al. ${ }^{21}$ sugerem que as SPCs podem ser desenvolvidas como um novo indicador citogenético relacionado à exposição, principalmente em avaliações do risco de câncer ocupacional, sendo sua utilização mais adequada para este tipo de análise. Os autores sugeriram, ainda, a condução de investigações epidemiológicas de estudos prospectivos e citogenéticos da relação das SPCs com a exposição às substâncias químicas utilizadas nos ambientes de trabalho ${ }^{21}$. A ideia de associação causal entre aberrações cromossômicas (numéricas e estruturais) e risco para câncer está baseada no conceito de que danos genéticos em linfócitos de sangue periférico refletem danos similares em células envolvidas nos processos carcinogênicos ${ }^{7}$. Portanto, a alta frequência de aneuploidias no grupo de trabalhadores do estaleiro indica a possibilidade de desenvolvimento de processos de carcinogenicidade.

\section{CONCLUSÕES}

Foram observadas alterações respiratórias e perda auditiva periférica de origem ocupacional no grupo de trabalhadores do estaleiro avaliado, com associação entre a PAIR e a concentração de chumbo em sangue. Os resultados indicam que a aneuploidia pode ser uma importante ferramenta na avaliação da citotoxicidade e exposição ocupacional a substâncias clastogênicas.

Deve ser realizado um programa para avaliação da exposição ocupacional nos estaleiros que permita orientar o estabelecimento de medidas que visem eliminar a exposição dos trabalhadores, como o emprego de processos automatizados, ou pelo menos limitar a exposição, melhorando a ventilação do local, a proteção respiratória individual e o uso de tintas seguras.

\section{REFERÊNCIAS}

1. Costa MFB, Costa MAF. Exposição ocupacional a compostos orgânicos voláteis na indústria naval. Quim Nova. 2002;25(3):384-6. http://dx.doi. org/10.1590/S0100-40422002000300009.

2. Morata TC. Chemical exposure as a risk factor for hearing loss. J Occup Environ Med. 2003;45(7):676-82. http://dx.doi.org/10.1097/01. jom.0000071507.96740.70. PMid:12855908.
3. Vyskocil A, Truchon G, Leroux T, Lemay F, Gendron M, Gagnon F, et al. A weight of evidence approach for the assessment of the ototoxic potential of industrial chemicals. Toxicol Ind Health. 2012;28(9):796-819. http:// dx.doi.org/10.1177/0748233711425067. PMid:22064681.

4. Morata TC, Lemasters G. Considerações epidemiológicas para o estudo de perdas auditivas ocupacionais. In: Nudelmann, A. A., Costa, E. A., 
Seligman, J., Ibañez, R. N. PAIR - Perda auditiva induzida pelo ruído. Rio de Janeiro: Revinter; 2001. Vol. 2, p. 1-16.

5. Demetriou CA, Raaschou-Nielsen O, Loft S, Møller P, Vermeulen R, Palli $\mathrm{D}$, et al. Biomarkers of ambient air pollution and lung cancer: a systematic review. Occup Environ Med. 2012;69(9):619-27. http://dx.doi.org/10.1136/ oemed-2011-100566. PMid:22773658.

6. Hagmar L, Strömberg U, Tinnerberg H, Mikoczy Z. The usefulness of cytogenetic biomarkers as intermediate endpoints in carcinogenesis. Int J Hyg Environ Health. 2001;204(1):43-7. http://dx.doi.org/10.1078/14384639-00071. PMid:11725344.

7. Bonassi S, Hagmar L, Strömberg U, Montagud AH, Tinnerberg H, Forni A, et al. Chromosomal aberrations in lymphocytes predict human cancer independently of exposure to carcinogens. Cancer Res. 2000;60(6):1619-25. PMid:10749131.

8. Rossner P, Boffetta P, Ceppi M, Bonassi S, Smerhovsky Z, Landa K, et al Chromosomal aberrations in lymphocytes of healthy subjects and risk of cancer. Environ Health Perspect. 2005;113(5):517-20. http://dx.doi. org/10.1289/ehp.6925. PMid:15866756.

9. Chandirasekar R, Kumar BL, Sasikala K, Jayakumar R, Suresh K, Venkatesan $\mathrm{R}$, et al. Assessment of genotoxic and molecular mechanisms of cancer risk in smoking and smokeless tobacco users. Mutat Res Genet Toxicol Environ Mutagen. 2014;767:21-7.http://dx.doi.org/10.1016/j.mrgentox.2014.04.007. PMid:24769293.

10. Valente D, Costa-Amaral IC, Carvalho LVB, Santos MVC, Castro VC, Rodrigues DRF, et al. Utilização dos biomarcadores de genotoxicidade e expressão gênica na avaliação de trabalhadores de postos de combustíveis expostos a vapores de gasolina. Rev Bras Saúde Ocup. 2016

11. Celi K A, Akbaş E. Evaluation of sister chromatid exchange and chromosomal aberration frequencies in peripheral blood lymphocytes of gasoline station attendants. Ecotoxicol Environ Saf. 2005;60(1):106-12. http://dx.doi. org/10.1016/j.ecoenv.2003.10.008. PMid:15482847.

12. Albertini RJ, Anderson D, Douglas GR, Hagmar L, Hemminki K, Merlo F, et al. IPCS guidelines for the monitoring of genotoxic effects of carcinogens in humans. International Programme on Chemical Safety. Mutat Res. 2000;463(2):111-72. http://dx.doi.org/10.1016/S1383-5742(00)00049-1. PMid:10913908

13. Miranda AC. A Vigilância em saúde na Indústria Naval: o caso dos trabalhadores de pintura em um estaleiro do Rio de Janeiro [dissertação]. Rio de Janeiro (RJ): Escola Nacional de Saúde Pública, Fundação Oswaldo Cruz; 1997.

14. Castro HA. Busca de marcadores inflamatórios IL-1ß, IL-6 e TNF a em trabalhadores expostos a poeiras minerais [tese]. Rio de Janeiro (RJ): Escola Nacional de Saúde Pública, Fundação Oswaldo Cruz; 2000.

15. Murata MM. Implementação da técnica de avaliação de aberrações cromossomiais e sua aplicação em indivíduos expostos a solventes e metais [dissertação]. Rio de Janeiro (RJ): Escola Nacional de Saúde Pública, Fundação Oswaldo Cruz; 2007.

16. Ramos TD. Avaliação da exposição ambiental ao manganês na população residente no entorno de um estaleiro no município de Angra dos Reis, RJ [dissertação]. Rio de Janeiro (RJ): Escola Nacional de Saúde Pública, Fundação Oswaldo Cruz; 2013.

17. Associação Brasileira de Normas Técnicas. NBR 10152: Níveis de ruído para conforto acústico. Rio de Janeiro: ABNT; 1987.
18. Instituto Nacional do Seguro Social. Ordem de Serviço/INSS/DAF/DSS n ${ }^{\circ}$ 608, de 05 de agosto de 1998. Aprova Norma Técnica sobre perda Auditiva Neurossensorial por Exposição Continuada a Níveis de Pressão Sonora de origem ocupacional. Brasília: INSS; 1998.

19. Neves EB, Mendonça Junior N, Moreira MF. Avaliação da exposição a metais numa oficina de recuperação de armamento de uma organização militar. Cien Saude Colet. 2009;14(6):2269-80. http://dx.doi.org/10.1590/ S1413-81232009000600036. PMid:20069197.

20. Mattos RCOC. Estratégias para avaliação da exposição ao chumbo: estudos comparativos dos indicadores biológicos e efeitos relacionados [tese de doutorado]. Rio de Janeiro (RJ): Instituto Oswaldo Cruz, Fundação Oswaldo Cruz; 2001

21. Major J, Jakab MG, Tompa A. The frequence of induced premature centrometre division in human populations occupationally exposed to genotoxic chemicals. Mutat Res. 1999;445(2):241-9. http://dx.doi.org/10.1016/ S1383-5718(99)00129-1. PMid:10575433.

22. Azevedo FA, Chasin AAM. Metais: gerenciamento da toxicidade. $1^{\text {a }}$ ed. São Paulo: Atheneu Inter Tox; 2003.

23. Skerfving S, Bergdhal IA. Lead. In: Nordberg GF, Fowler BA, Nordberg M, Friberg LT, editors. Handbook on the Toxicology of Metals. $3^{a}$ ed. USA: Academic Press; 2007. p. 599-643.

24. Pelclová $\mathrm{D}$, Cerná $\mathrm{M}$, Pastorková $\mathrm{A}$, Vrbíková V, Procházka B, Hurychová D, et al. Study of the genotoxicity of toluene. Arch Environ Health. 2000;55(4):268-73. http://dx.doi.org/10.1080/00039890009603417. PMid:11005432.

25. Holecková B, Piesová E, Sivikova K, Dianovskỳ J. Chromosomal aberrations in humans induced by benzene. Ann Agric Environ Med. 2004;11(2):175-9. PMid:15627321.

26. Villalba-Campos M, Chuaire-Noack L, Sánchez-Corredor MC, RondónLagos M. High chromosomal instability in workers occupationally exposed to solvents and paint removers. Mol Cytogenet. 2016;9(1):46. http://dx.doi. org/10.1186/s13039-016-0256-6. PMid:27325915.

27. Silva TF, Rodrigues DRF, Coutinho GBF, Soares AMT, Almeida MS, Sarcinelli PN, et al. Ototoxicity of hydrocarbons present in gasoline: a literature review. Rev CEFAC. 2018;20(1):110-21.

28. Brasil. Ministério da Saúde. Secretaria de Atenção à Saúde. Departamento de Ações Programáticas Estratégicas. Risco químico: atenção à saúde dos trabalhadores expostos ao benzeno. Brasília: Editora do Ministério da Saúde; 2006. 48 p. (Série A. Normas e Manuais Técnicos; Saúde do Trabalhador; 7). (Protocolos de Complexidade Diferenciada).

29. Brasil. Ministério da Saúde. Secretaria de Atenção à Saúde. Departamento de Ações Programáticas Estratégicas. Câncer relacionado ao trabalho: leucemia mielóide aguda - síndrome mielodisplásica decorrente da exposição ao benzeno. Brasília: Editora do Ministério da Saúde; 2006. 48 p. (Série A. Normas e Manuais Técnicos; Saúde do Trabalhador; 8). (Protocolos de Alta Complexidade).

30. Alvarenga KF, Jacob LCB, Martins CHF, Costa OA, Coube CZV, Marques JM. Emissões otoacústicas - produto de distorção em indivíduos expostos ao chumbo e ao ruído. Rev Bras Otorrinolaringol. 2003;69(5):681-9. http:// dx.doi.org/10.1590/S0034-72992003000500014.

31. Teixeira CF, Augusto LG, Morata TC. Saúde auditiva de trabalhadores expostos a ruído e inseticidas. Rev Saude Publica. 2003;37(4):417-23. http://dx.doi.org/10.1590/S0034-89102003000400005. PMid:12937701. 
32. Botelho CT, Paz APML, Gonçalves AM, Frota S. Estudo comparativo de exames audiométricos de metalúrgicos expostos a ruído e ruído associado a produtos químicos. Rev Bras Otorrinolaringol. 2009;75(1):51-7. http:// dx.doi.org/10.1590/S0034-72992009000100008.

33. Lima ERZ, Colon JC, Souza MT. Alterações auditivas em trabalhadores expostos a mercúrio. Rev CEFAC. 2009;11(Supl 1):62-7.

34. Guida HL, Morini RG, Cardoso ACV. Avaliação audiológica em trabalhadores expostos a ruído e praguicida. Rev Bras Otorrinolaringol. 2010;76(4):423-7.

35. Leão RN, Dias FAM. Perfil audiométrico de indivíduos expostos ao ruído atendidos no núcleo de saúde ocupacional de um hospital do município de Montes Claros, Minas Gerais. Rev CEFAC. 2010;12(2):242-9. http:// dx.doi.org/10.1590/S1516-18462010000200010.

36. Ferreira DG, Oliveira GL, Meira AL, Lacerda A. Efeitos auditivos da exposição combinada: interação entre monóxido de carbono, ruído e tabagismo. Rev Soc Bras Fonoaudiol. 2012;17(4):405-11. http://dx.doi. org/10.1590/S1516-80342012000400007.

37. Quevedo LS, Tochetto T, Siqueira MA, Machado MS. Potenciais evocados auditivos de tronco encefálico em frentistas. Braz J Otorhinolaryngol. 2012;78(6):63-8.

38. Quevedo LS, Tochetto T, Siqueira MA. Condição coclear e do sistema olivococlear medial de frentistas de postos de gasolina expostos a solventes orgânicos. Arq Int Otorrinolaringol. 2012;16(1):50-6.

39. Tochetto TM, Quevedo LS, Siqueira MA. Condição auditiva de frentistas. Rev CEFAC. 2013;15(5):1137-47.

40. Alcarás PA, Larcerda AB, Marques JM. Study of evoked otoacoustic emissions and suppression effect on workers exposed to pesticides and noise. CoDAS. 2013;25(6):527-33. PMid:24626978.

41. Haeffner R, Sarquis LM, Heck RM, Jardim VM. Prevalence of hearing problems and associated factors in an agricultural company in southern Brazil. Rev Bras Epidemiol. 2015;18(3):679-90. http://dx.doi.org/10.1590/19805497201500030013. PMid:26247191.

42. Seligman J. Sintomas e sinais na PAIR. In: Nudelmann, AA, Costa, EA, Seligman, J, Ibañez, RN, organizadores. PAIR: perda auditiva induzida pelo ruído. Porto Alegre: Baggagem; 1997. p. 143-51.

43. Souza MMN, Bernardi APA. Ototoxidade dos produtos químicos: enfoque ocupacional. Rev CEFAC. 2001;3:95-102.

44. Jacob LCB, Alvarenga KF, Morata TC. Os efeitos da exposição ocupacional ao chumbo sobre o sistema auditivo: uma revisão da literatura. Rev Bras Otorrinolaringol. 2002;68(4):564-9. http://dx.doi.org/10.1590/S003472992002000400018

45. Brasil. Ministério da Saúde. Secretaria de Atenção à Saúde. Departamento de Ações Programáticas Estratégicas. Atenção à saúde dos trabalhadores expostos ao chumbo metálico. Brasília: Editora do Ministério da Saúde, 2006. 48 p. (Série A. Normas e Manuais Técnicos; Saúde do Trabalhador; 4). (Protocolos de Complexidade Diferenciada).

46. Brasil. Ministério do Trabalho e Emprego. Portaria GM n. ${ }^{\circ} 3.214$, de 08 de junho de 1978. NR 7 - Programa de Controle Médico de Saúde Ocupacional. Diário Oficial da União.

47. Occupational Safety and Health Administration. Chemical Sampling Information: Lead, Inorganic (as Pb) [Internet]. 2015 [citado em 2015 junho 5]. Disponível em: https://www.osha.gov/dts/chemicalsampling/ data/CH_249110.html
48. American Conference of Governmental Industrial Hygienists. TLVs ${ }^{\oplus} \mathrm{e}$ BEIs $^{\circ}$ - American Conference of Governmental Industrial Hygienists (ACGIH). Associação Brasileira de Higienistas Ocupacionais (ABHO), Tradução. São Paulo: ABHO; 2009. 287.

49. National Institute for Occupational Safety and Health. Worker health chartbook, 2000. Washington: U.S. Department of Health and Human Services, Public Health Service, Centers for Disease Control and Prevention, National Institute for Occupational Safety and Health; 2000. Publication Number 2000-127.

50. Apostoli P, Lucchini R, Alessio L. Are current biomarkers suitable for the assessment of manganese exposure in individual workers? Am J Ind Med. 2000;37(3):283-90. http://dx.doi.org/10.1002/(SICI)10970274(200003)37:3<283::AID-AJIM6>3.0.CO;2-E. PMid:10642418.

51. Minoia C, Sabbioni E, Apostoli P, Pietra R, Pozzoli L, Gallorini M, et al. Trace element reference values in tissues from inhabitants of the European community. I. A study of 46 elements in urine, blood and serum of Italian subjects. Sci Total Environ. 1990;95:89-105. http://dx.doi.org/10.1016/00489697(90)90055-Y. PMid:2402627.

52. U.S. Department of Health and Human Services, Public Health Service, Agency for Toxic Substances and Disease Registry. Toxicological profile for manganese. Washington: 2008.

53. Moreira MF, Neves EB. Uso do chumbo em urina como indicador de exposição e sua relação com chumbo no sangue. Cad Saude Publica. 2008;24(9):2151-9. http://dx.doi.org/10.1590/S0102-311X2008000900021. PMid:18813691.

54. World Health Organization, International Agency for Research on Cancer. IARC Monographs on the Evaluation of Carcinogenic Risks to Humans [Internet]. 2014 [citado em 2014 maio 5]. Disponível em: http://monographs. iarc.fr/ENG/Classification/

55. Ferrari I, Gonçalves A, Barbosa A, Centeno AJ, Padovani CR, Gonçalves NNS. Investigação de genotoxicidade do mercúrio em pessoas profissionalmente expostas em garimpo de região da Amazônia Legal II-resultados dosimétricos e genotóxicos. Rev Bras Saúde Ocup. 1992;76(20):10-4.

56. Santos-Mello R, Cavalcante B. Cytogenetic studies on gas station attendants. Mutat Res. 1992;280(4):285-90. http://dx.doi.org/10.1016/0165-1218(92)900599. PMid:1382230.

57. Bréga SM, Vassilieff I, Almeida A, Mercadante A, Bissacot D, Cury PR, et al. Clinical, cytogenetic and toxicological studies in rural workers exposed to pesticides in Botucatu, São Paulo, Brazil. Cad Saude Publica. 1998;14(Suppl 3):S109-15. http://dx.doi.org/10.1590/S0102-311X1998000700011. PMid:9819469.

58. Estecio MR, Silva AE. Alterações cromossômicas causadas pela radiação dos monitores de vídeo de computadores. Rev Saude Publica. 2002;36(3):330-6. http://dx.doi.org/10.1590/S0034-89102002000300012. PMid:12131973.

59. Miyaji CK, Colus IM. Cytogenetic biomonitoring of Brazilian dentists occupationally exposed to low doses of X-radiation. Pesqui Odontol Bras. 2002;16(3):196-201. http://dx.doi.org/10.1590/S1517-74912002000300003. PMid:12386679.

60. Chinelato AR, Froes NDTC. Genotoxic effects on professionals exposed to inhalational anesthetics. Rev Bras Anestesiol. 2002;52(1):79-85.

61. Santos MFA, Ferrari I, Luna H. Chromosomal aberration analysis in workers exposed to chemical and biological hazards in research laboratories. Environ Res. 2005;97(3):330-4. http://dx.doi.org/10.1016/j.envres.2004.09.013. PMid:15589242. 
62. Goncalves RO, Melo NA, Carvalho FM, Goes RC. Efeitos genotóxicos e alterações de enzimas hepáticas em trabalhadores do refino de petróleo. J Bras Patol Med Lab. 2005;41(5):297-9.

63. Silva-Barbosa I, Pereira-Magnata S, Amaral A, Sotero G, Melo HC. Dose assessment by quantification of chromosome aberrations and micronuclei in peripheral blood lymphocytes from patients exposed to gamma radiation. Genet Mol Biol. 2005;28(3):452-7. http://dx.doi.org/10.1590/ S1415-47572005000300021.

64. Trevisan P, da Silva JN, da Silva AP, Rosa RF, Paskulin GA, Thiesen FV, et al. Evaluation of genotoxic effects of benzene and its derivatives in workers of gas stations. Environ Monit Assess. 2014;186(4):2195-204. http://dx.doi. org/10.1007/s10661-013-3529-0. PMid:24292950.

65. Santiago F, Alves G, Otero UB, Tabalipa MM, Scherrer LR, Kosyakova $\mathrm{N}$, et al. Monitoring of gas station attendants exposure to benzene, toluene, xylene (BTX) using three-color chromosome painting. Mol Cytogenet. 2014;7:15. http://dx.doi.org/10.1186/1755-8166-7-15. PMid:24576355.

Recebido em: Out. 25, 2016

Aprovado em: Out. 28, 2017 